\title{
Editorial
}

\section{Transition from Microscopic to Endoscopic Pituitary Surgery}

\author{
Abrar Ahad Wani ${ }^{1}$ Mohsin Bhat ${ }^{1}$ \\ ${ }^{1}$ Department of Neurosurgery, Sher-i-Kashmir Institute of Medical \\ Sciences, Srinagar, Jammu and Kashmir, India \\ Indian J Neurosurg 2019;8:91-92
}

The microscopic transnasal tumor surgery (TNTS) has stood the test of time since its inception in 1960; however, the advent of the endoscopic nasal pituitary surgery (EPS) has made inroads in this particular field with its myriad benefits. Both TNTS and EPS procedures are effective tools in tackling pituitary tumors. ${ }^{1}$ The ardent supporters of TNTS promote the benefits of maintaining stereoscopic vision, instrument mobility, control of bleeding effectively, retraction of the edematous nasal tissues, and quick access to the sella. On the other hand, the transition to the EPS procedure is happening worldwide, clearly on account of its advantages, which include a wide panoramic view, improved illumination, ability to look around the anatomical corners using an angled tip, and extended approach to the paraseller tumors. ${ }^{1}$ Instrumental maneuverability during the endoscopic procedures has been alleviated with the introduction of newer endoscopic specific instruments, and high-definition 3D endoscopic system may resolve issues pertaining to stereoscopic resolution.

Meta-analyses reveal a clear advantage of EPS over the TNTS procedure in relation to the locally invasive tumor with parasellar extension; its benefits also include more effective resection and good endocrinological outcome. ${ }^{2,3}$ For tumors invading the cavernous sinus, higher resection rates have been observed, with the endoscope depicting a clear advantage of the panoramic and the angled views of the cavernous sinus medial wall. ${ }^{4,5}$ EPS is considered a valid option in treating recurrent forms of adenoma. Wide exposure of the sella is especially ensured, keeping in mind the limited exposure of the previous microscopic surgical procedures, which was responsible for incomplete tumor resection. Nasal morbidities such as sinusitis, anosmia, epistaxis, nasal congestion, and septal perforation are comparatively lesser in amount in the endoscopic nasal surgery. ${ }^{1}$ A comfortable postoperative course is provided by way of the following:

1. Lesser mucosal detachment

2. Absence of sub-labial incision

3. Absence of routine nasal packing

Address for correspondence Abrar Ahad Wani, MCh, Department of Neurosurgery, Sher-i-Kashmir Institute of Medical Sciences, Soura, Srinagar 190011, J\&K, India (e-mail: drabrarahadwani@gmail.com).
These result in the reduction of the postoperative pain and ensure a quick recovery and short hospital stay. ${ }^{1}$

Despite the wider exposure and dissection, and the attendant higher risk of the intra- operative CSF leak, the incidence of the postoperative leak in the EPS is equivalent to the microscopic surgery in the recent literature. ${ }^{1}$ The risk of permanent diabetes insipidus is between 0.7 and $8.5 \%{ }^{6}$ Injury to the carotid artery is a rare but potentially life-threatening complication. ${ }^{7}$ Thus, in general, the surgical complication rate of the endoscopic approach is equal to that of the microscopic approach. Endoscopic nasal surgery is cost effective in comparison to its microscopic counterpart. The learning curve may deter some microscopically trained neurosurgeons from embracing the endoscopic approach, because of an unwillingness on their part to accept the complications during the procedure. This can be overcome by attending workshops concerning endoscopic nasal and paranasal anatomy, endoscopic surgery, and then gradually moving from endoscopic-assisted microscopic procedure to pure EPS. In the initial cases, proper case selection, operative planning, instrumentation with assistance from a surgeon well-versed with the technique would help to make a tangible difference. Also, an attitude in favor of converting EPS to TNTS immediately in case of difficulty would be a good approach to avert any complication.

Endoscopic nasal pituitary surgery with its innovative techniques such as 3D endoscopic system, endoscopic augmented reality navigation system, and ultrasonography-assisted endoscope have increased the safety and revolutionized the concept of pituitary surgeries in developed countries. ${ }^{1}$ Despite their relative absence in our part of the world, the results are extremely encouraging.

The EPS is definitely going to replace TNTS, and it is only the time that will decide when, as a famous quote from Steve Jobs says,

"The over-all point is that new technology will not necessarily replace old technology, but it will date it. By definition. Eventually, it will replace it. But it is like people who had

(c)2019 Neurological Surgeons' Society License terms

of India

() (1) $\Theta \circledast$ 10.1055/s-0039-1697529

ISSN 2277-954X. 
black-and-white TVs when color came out. They eventually decided whether or not the new technology was worth the investment."

\section{Conflicts of Interest}

None declared.

\section{References}

1 Nishioka H. Recent Evolution of Endoscopic Endonasal Surgery for Treatment of Pituitary Adenomas. Neurol Med Chir (Tokyo) 2017;57(4):151-158

2 Singh H, Essayed WI, Cohen-Gadol A, Zada G, Schwartz TH. Resection of pituitary tumors: endoscopic versus microscopic. J Neurooncol 2016;130(2):309-317

3 Gao Y, Zhong C, Wang Y, et al. Endoscopic versus microscopic transsphenoidal pituitary adenoma surgery: a meta-analysis. World J Surg Oncol 2014;12:94
4 Ceylan S, Koc K, Anik I. Endoscopic endonasal transsphenoidal approach for pituitary adenomas invading the cavernous sinus. J Neurosurg 2010;112(1):99-107

5 Dhandapani S, Singh H, Negm HM, Cohen S, Anand VK, Schwartz TH. Cavernous sinus invasion in pituitary adenomas: systematic review and pooled data meta-analysis of radiologic criteria and comparison of endoscopic and Microscopic surgery. World Neurosurg 2016;96:36-46

6 Messerer M, De Battista JC, Raverot G, et al. Evidence of improved surgical outcome following endoscopy for nonfunctioning pituitary adenoma removal. Neurosurg Focus 2011;30(4):E11

7 Tabaee A, Anand VK, Barrón Y, et al. Endoscopic pituitary surgery: a systematic review and meta-analysis. J Neurosurg 2009;111(3):545-554 